\title{
Radiative cooling effects on oscillating loops
}

\section{César A. Mendoza-Briceño and Marialejandra Luna-Cardozo}

Centro de Física Fundamental, CFF, Facultad de Ciencias, Universidad de Los Andes, La Hechicera, Mérida 5251, Venezuela, email: cesar@ula.ve

\begin{abstract}
Recently, high resolution observations by $S O H O$ and TRACE spacecraft have identified oscillating loops and propagating waves in the solar coronal. These new discoveries established a new discipline that is known as coronal seismology. The importance of this lies in the potential for the diagnostics of coronal structures and knowledge of coronal heating. We present a study of the effects of radiative cooling and heating processes on longitudinal waves in coronal loops. We find that radiation and heating results in a clear modification in the evolution of temperature and pressure perturbations but in slight effect on the decay time of the wave.
\end{abstract}

Keywords. Sun: corona, hydrodynamics, Sun: oscillations

\section{Introduction}

Recent observations by high-resolution space imaging telescopes and spectrometers have revealed a variety of coronal oscillation modes. Such oscillations are important because of their potential for the diagnostics of coronal structures (magnetic field strength, gas density, etc.) through coronal seismology and their potential for heating the corona (Roberts \& Nakariakov 2003).

Since the plasma dynamics in a coronal loop is dominated by the magnetic field, we make the usual assumption that the plasma motion takes place primarily along the magnetic field lines which in turn determine the loop geometry. The energy conservation equation reads

$$
\rho \frac{d T}{d t}=-\frac{\bar{\mu}(\gamma-1)}{\mathcal{R}_{g}}\left[p \frac{\partial v}{\partial s}+\rho^{2} Q(T)-H_{0}-\frac{\partial}{\partial s}\left(\kappa \frac{\partial T}{\partial s}\right)-\frac{4}{3} \eta\left(\frac{\partial v}{\partial s}\right)^{2}\right],
$$

where $t$ is time, $s$ denotes the position along a loop of constant cross-section, $\rho$ is the plasma mass density, $v$ is the fluid velocity, $T$ is the plasma temperature, $p$ is the gas pressure, $Q(T)=\chi T^{\alpha}$ is the optically thin radiation-loss function (Cook et al. 1989), $H_{0}$ is the constant coronal heating function, $\gamma(=5 / 3)$ is the ratio of specific heats, $\mu$ is the mean molecular weight, $\kappa=10^{-6} T^{5 / 2} \mathrm{ergs} \mathrm{cm}^{-1} \mathrm{~s}^{-1} \mathrm{~K}^{-1}$ is the coefficient of thermal conductivity parallel to the magnetic field, and $\eta$ is the coefficient of compressive viscosity (Braginskii 1965). Equation (1.1) together with continuity and momentum equations are closed by assuming a pressure relation of the form $p=\mathcal{R}_{g} \rho T / \mu$, where $\mathcal{R}_{g}$ is the gas constant. The set of governing equations are solved numerically using a $1 \mathrm{D}$, finitedifference (FD) hydrodynamics code based on a temporally and spatially second-order accurate Lagrangian remap technique (Sigalotti \& Mendoza-Briceño 2003).

We choose a one-dimensional loop configuration of semicircular shape, constant crosssectional area, and total length $L=400 \mathrm{Mm}$, with an initial uniform temperature $(T=$ 6.3 or $8.0 \mathrm{MK})$ and density $\left(=5.0 \times 10^{8} \mathrm{~cm}^{-3}\right)$ distributions, and the initial velocity is of the form $v=v_{0} \sin (2 \pi s / L)$, where $v_{0}$ is the amplitude of the wave at $t=0$. As outlined by Mendoza-Briceño et al. (2004), these initial parameters are motivated by SUMER and Yohkoh SXT observations of hot loops in the upper solar atmosphere. 

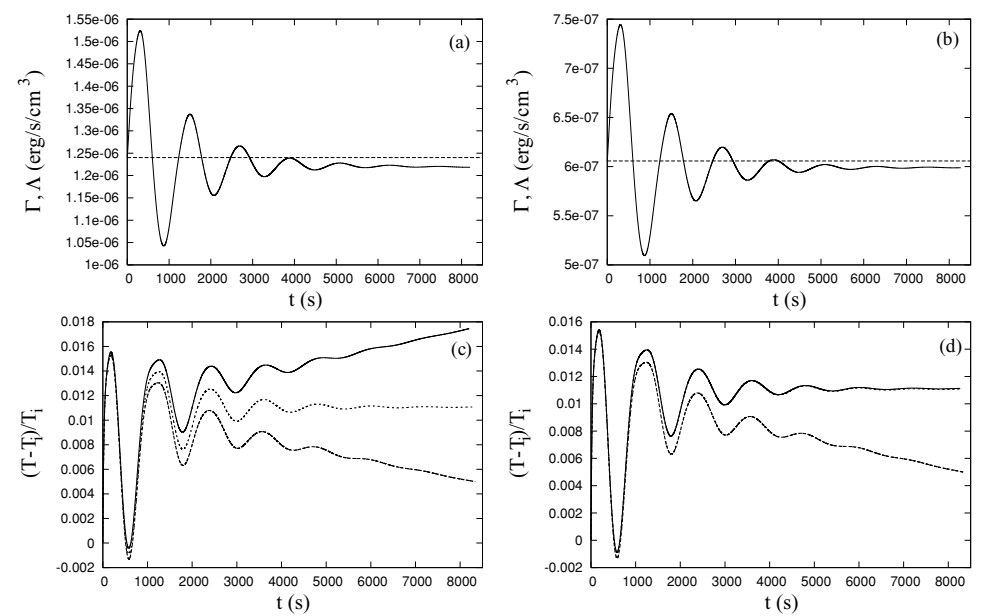

Figure 1. Time evolution of the cooling (solid line) and heating (dashed line) processes (top panels) for a loop model with (a) $T=6.3 \mathrm{MK}$ and (b) $T=8.0 \mathrm{MK}$. Time evolution of the temperature at $s=0.35 \mathrm{~L}$ for a loop model with (c) $T=6.3 \mathrm{MK}$ and (d) $T=8.0 \mathrm{MK}$. The wave evolution including cooling and heating (solid line) is compared at the same evolution when only cooling is considered (dashed line), and when the cooling and heating is neglected (dotted line).

\section{Results and Discussion}

In this paper we extend the 1D loop model calculations of Mendoza-Briceño et al. (2004) to include the effects of radiative cooling and heating processes on damping of longitudinal waves in hot coronal loops. In Figure 1, we display the cooling and heating processes as a function of time for an initial temperatures of (a) $T=6.3 \mathrm{MK}$ and (b) $T=8.0 \mathrm{MK}$. The resulting time evolution of the temperature perturbation at a fixed distance $l_{0}=0.35 \mathrm{~L}$ for $T=6.3 \mathrm{MK}$ and $T=8.0 \mathrm{MK}$ are shown in Figures $1 \mathrm{c}$ and $1 \mathrm{~d}$, respectively. It is observed that the cooling and heating processes affect primarily the temperature and pressure perturbations. We find that including only radiative losses makes the temperature and pressure perturbations to decrease in time. For the case of $T=6.3 \mathrm{MK}$ the temperature perturbation increases in time when both the cooling and heating are considered, as well as the pressure perturbation. This is believed to be caused by the heating that is slightly higher that the cooling after two periods. Increasing the initial temperature to $T=8.0 \mathrm{MK}$, the evolution of the temperature and pressure perturbations show no variations when both processes are included and it is compared to the case without these two effects. It is probably due to the fact the heating decreases to half of the value for the case when $T=6.3 \mathrm{MK}$, and the loop seems to be evolving as it were in thermal equilibrium.

\section{Acknowledgements}

CAM-B would like to thank the CDCHT of the Universidad de Los Andes for financial support (C-1367-06-05-B). ML-C acknowledges support by the FONACIT.

\section{References}

Braginskii, S.I. 1965, Rev.Plasma Phys. 1, 205

Cook, J.W., Cheng, C.-C., Jacobs, V.L., \& Antiochos, S.K. 1989, ApJ 338, 1176

Mendoza-Briceño, C.A., Erdélyi, R. \& Sigalotti, L.G. 2004, ApJ 605, 493

Roberts, B. \& Nakariakov, V.M. 2003, NATO Science Series II 124, 167

Sigalotti, L. Di G. \& Mendoza-Briceño, C. A. 2003, A\&\&A 397, 1083 